\title{
LAS ASAMBLEAS DE PROFESORES EN LA CONSOLIDACIÓN DEL CONSEJO DE ENSEÑANZA SECUNDARIA EN URUGUAY (1949-1961)
}

DOI: http://dx.doi.org/10.1590/2236-3459/80720

\author{
Lucas D'Avenia' \\ 'Universidad de la República (Udelar), Montevideo, Uruguay
}

$\cos 80$

\begin{abstract}
Resumen
El artículo explora la creación y la primera etapa de funcionamiento de las Asambleas de Profesores del Consejo de Enseñanza Secundaria entre 1949 y 1961. Las asambleas fueron creadas para discutir los asuntos técnico-pedagógicos relacionados con la enseñanza secundaria y asesorar a las autoridades en la toma de decisiones. Del análisis de las fuentes se desprende que en el período estudiado las asambleas de profesores acompañaron la consolidación institucional del Consejo que había sido creado en 1935 y ampliaron el protagonismo de los profesores que se convirtieron en un actor central de las discusiones pedagógicas sobre la enseñanza media en un período de intenso debate.

Palabras clave: enseñanza secundaria, Uruguay, profesores.
\end{abstract}

\section{ASSEMBLÉIAS DE PROFESSORES NA CONSOLIDAÇÃO DO CONSELHO DE ENSINO SECUNDÁRIO NO URUGUAI (1949-1961)}

\begin{abstract}
Resumo
O artigo explora a criação e a primeira etapa de funcionamento das Assembléias de Professores do Conselho de Ensino Secundária entre 1949 e 1961. As assembléias foram criadas para discutir as questões técnicopedagógicas relacionadas ao ensino secundário e aconselhar as autoridades na tomada de decisões. A partir da análise das fontes, pode-se ver que, durante o período estudado, as assembléias de professores acompanharam a consolidação institucional do Conselho, que havia sido criado em 1935. Além disso, também expandiram o papel dos professores que se tornaram um ator central nas discussões pedagógicas sobre 0 ensino médio em um período de intenso debate.

Palavras-chave: ensino médio, Uruguay, professores.
\end{abstract}

\section{ASSEMBLIES OF TEACHERS IN THE CONSOLIDATION OF THE SECONDARY EDUCATION COUNCIL IN URUGUAY (1949-1961)}

\begin{abstract}
The article explores the creation and the first stage of operation of the Teachers' Assemblies of the Secondary School Council between 1949 and 1961. The assemblies were created to discuss the technical-pedagogical issues related to secondary education and advise the authorities in the decision taking process. From the analysis of the sources, it can be inferred that in the period studied, the teachers' assemblies accompanied the institutional consolidation of the Council that had been created in 1935 and expanded the role of the teachers who became a central actor in the pedagogical discussions on secondary education in a period of intense
\end{abstract}


debates.

Keywords: secondary education, Uruguay, teachers.

\section{ASSEMBLÉES D'ENSEIGNANTS À LA CONSOLIDATION DU CONSEIL DE L'ÉDUCATION SECONDAIRE EN URUGUAY (1949-1961)}

\section{Résumé}

L'article explore la création et la première étape des Assemblées des Enseignants du Conseil de Enseignement Secondaire entre 1949 et 1961. Les assemblées ont été créées pour discuter des questions techniques et pédagogiques liées à l'enseignement secondaire et pour conseiller les autorités dans la prise de decisions. De l'analyse des sources, on peut déduire que pendant la période étudiée les assemblées d'enseignants ont accompagné la consolidation institutionnelle du Conseil, qui avait été créée en 1935. Elles ont également élargi le rôle des enseignants qui sont devenus un acteur central dans les discussions pédagogiques à propos de l'enseignement secondaire dans une période de débat intense.

Mots-clés: enseignement secondaire, Uruguay, enseignants. 


\section{Presentación}

A partir de 1949, en el organismo a cargo de la enseñanza media pública en Uruguay, el Consejo Nacional de Enseñanza Secundaria, funcionó una instancia orgánica de participación de los docentes: la Asamblea de Profesores. ${ }^{1}$ Las Asambleas estaban orientadas a discutir los asuntos técnico-pedagógicos relacionados con la enseñanza secundaria para asesorar a las autoridades en la toma de decisiones. Este trabajo se detiene en el proceso de conformación de este ámbito institucional. Nos proponemos caracterizar los antecedentes de dicha institucionalidad, describir el proceso a través del cual las asambleas de profesores se consolidaron como dispositivo de producción de discursos pedagógicos y analizar su agenda entre 1949 y 1961.

Las Asambleas de Profesores en el ámbito de la enseñanza secundaria uruguaya fueron un dispositivo peculiar. Si bien pueden tener parecidos de familia con otras estructuras colectivas de circulación de ideas - como congresos, asociaciones profesionales y organizaciones gremiales -, las Asambleas tuvieron características propias. En ellas se combinaron las lógicas de la representación, de la deliberación y de la producción de discurso pedagógico. Pero además tuvieron una existencia formal, como resultado de una disposición legal ${ }^{2}$.

Algunos trabajos han planteado el papel de las Asambleas en la formación de una nueva "cultura institucional" (ROMANO, 2010, p. 41) que se desarrolló en torno a una serie de propuestas de reforma que procuraban responder a diagnósticos de crisis e inadecuación de la enseñanza a la realidad contemporánea. Otros estudios han incluido a las Asambleas en el proceso de organización sindical de los docentes de Secundaria. Estas instancias de reunión habrían sido ámbitos fundamentales para creación de la Gremial de Profesores de Montevideo en 1963 y la Federación Nacional de Profesores en $1964 .^{3}$ (YAFFÉ, 2006).

El estudio de la documentación emanada de las asambleas ${ }^{4}$ permite, en primer

\footnotetext{
${ }^{1}$ Este trabajo es resultado del proyecto de investigación "La irrupción del desarrollismo en las propuestas de reforma educativa en el Uruguay entre 1961 y 1967" financiado por la Comisión Sectorial de Investigación Científica de la Universidad de la República y llevado a cabo en el Instituto de Educación de la Facultad de Humanidades y Ciencias de la Educación de la Universidad de la República, Uruguay bajo la dirección de Antonio Romano. Una versión anterior fue presentada en el simposio "Intelectuales de la educación en América Latina: trayectorias, redes e intercambios (siglos XIX y XX) del IV Congreso Internacional de Ciencias, Tecnologías y Culturas, Universidad de Santiago de Chile, 2015.

2 Ley 10973 aprobada por la Asamblea General del Poder Legislativo el 20 de noviembre de 1947.

3 La Gremial de Profesores de Montevideo se constituyó formalmente en 1963. El grupo que promovió su organización compartió desde 1960 otras instancias colectivas en que se manifestó el descontento con la situación del organismo: la Asamblea Intergremial de docentes y administrativos que llevó adelante un conflicto por presupuesto en 1960 y la lista Nueva Secundaria que compareció en las elecciones del Consejo. La creación de la Gremial significó una ruptura con la Asociación de Profesores de Enseñanza Secundaria y Preparatoria que existía desde 1919, había apoyado la separación de Secundaria de la Universidad en 1935, tenía un perfil de asociación profesional más que sindical y se la asociaba generalmente a las políticas llevadas a cabo desde el Consejo. La Asamblea Intergremial de 1960, con la posición contraria de la Asociación de Profesores, llevó a cabo una huelga por primera vez en Secundaria. La Federación Nacional de Profesores se terminó de conformar en 1964 y tuvo la peculiaridad de reunir en una única estructura a todas las asociaciones gremiales de Secundaria que tenían alcance local o regional, en un régimen federativo. (YAFFÉ, 2006).

${ }^{4}$ Se accedió a un conjunto variado e incompleto de documentos relativos a las Asambleas de Profesores. Se trata de informes que servían de insumos para la discusión, documentos de comisiones temáticas de las
} 
lugar, caracterizar un ámbito de producción intelectual estrechamente conectado con la vida institucional del sistema educativo y los procesos de decisión de política educativa, ya que las asambleas fueron un ámbito de intercambio de ideas sobre la enseñanza, en el seno del organismo que estaba a cargo de su administración. Este intercambio se produjo en un contexto de intensas discusiones sobre la necesidad de reformar la enseñanza media, por lo tanto el trabajo busca avanzar en la comprensión de las ideas acerca de los problemas y las posibilidades de cambio que tenían los actores docentes. Además, las asambleas muestran un aspecto relevante de la institucionalización del Consejo de Enseñanza Secundaria, que había sido creado en 1935. Por un lado, evidencian el esfuerzo por instalar una cultura institucional en que los docentes debían tener protagonismo en la elaboración de las políticas educativas. Pero al mismo tiempo, permiten ver los esfuerzos por distinguir las particularidades de la enseñanza secundaria respecto del sistema educativo y de su inscripción en la sociedad en el período.

Además de describir el proceso mediante el cual las Asambleas se convirtieron en un escenario central en Secundaria y la agenda de política educativa que desplegaron, el artículo avanza en torno a otras interrogantes. ¿Cuál fue la magnitud que efectivamente tuvieron las Asambleas de Profesores? ¿Qué condiciones favorecieron que se convirtiera en un espacio central de elaboración pedagógica? ¿Por qué la elaboración programática y discusión conceptual del profesorado se canalizó mediante un dispositivo de este tipo? ¿Cómo se inscribió en la tradición uruguaya de autonomías del sistema educativo?

\section{La reconfiguración institucional de la enseñanza secundaria en Uruguay}

La enseñanza secundaria pública en Uruguay estuvo a cargo de la Sección de Enseñanza Secundaria y Preparatoria de la Universidad de la República hasta 1935. La Sección era dirigida por un Consejo Directivo al igual que las demás Facultades. Mediante una ley ${ }^{5}$ aprobada como resultado de un acuerdo entre el sector herrerista del Partido Nacional y el sector terrista del Partido Colorado se creó durante la dictadura encabezada por el presidente Gabriel Terra, un ente autónomo dirigido por el Consejo Nacional de Enseñanza Secundaria ${ }^{6}$.

Si bien la reorganización del nuevo Consejo implicó un proceso de construcción institucional, éste no debe ser considerado como una creación ex nihilo. Secundaria tenía una existencia propia dentro de la estructura universitaria, en buena medida porque el conjunto de la Universidad tenía una forma de funcionar que se ha denominado como federativa, donde cada facultad tenía importantes márgenes de autonomía y las acciones centralmente orientadas eran escasas ${ }^{7}$. Se sumaba a esta realidad general, el carácter

asambleas, documentos aprobados por el plenario, documentos de la Comisión Permanente y sus subcomisiones que actuaron entre una y otra convocatoria, y materiales de diverso tipo. En algunos casos se trata de materiales especialmente publicados en boletines para su difusión entre el profesorado. Se ha accedido a ellos a través del archivo personal del Inspector Arturo Rodríguez Zorrilla y a través de otras donaciones, disponibles en el Instituto de Educación de la Facultad de Humanidades y Ciencias de la Educación de la Universidad de la República, Uruguay.

5 Ley 9523, aprobada por la Asamblea General del Poder Legislativo el 9 de diciembre de 1935.

6 Ver Consejo de Educación Secundaria (2008), Oddone; Paris (2010).

7 Esta es una de las críticas realizadas al funcionamiento universitario que se derivó de la Ley Orgánica de 1908 (MARKARIAN; JUNG; WSCHEBOR, 2008b). A diferencia de la autonomía del Poder Ejecutivo y del cogobierno de los tres órdenes, este aspecto no fue sustancialmente modificado con la Ley Orgánica de 
específico de la enseñanza secundaria y las preocupaciones respecto a su naturaleza, que anteceden a 1935. Sin embargo, el período posterior a la creación del Consejo de Enseñanza Secundaria como ente autónomo, implicó una reorganización administrativa, pero también una reorientación en términos de identidad institucional. No sin fuertes tensiones, este proceso tuvo algunos hitos en la década siguiente.

La aprobación del Estatuto del Profesor, junto con la creación del Instituto de Profesores y la constitución de un Escalafón docente, fueron los pilares en que se fundó la nueva institucionalidad del Consejo Nacional de Enseñanza Secundaria. El Instituto de Profesores estaba previsto en una ley de $1937^{8}$, pero su creación se dispuso finalmente con la ley de presupuesto de $1949^{9}$ y sus actividades iniciaron en 1951. Pese al bajo número de egresos en los primeros años el Instituto fue una respuesta a la necesidad creciente de formación de los cuadros docentes. En 1947 el Parlamento aprobó la ley 10.973 que contenía el Estatuto del Profesor que regulaba el desempeño de la función pública de los docentes de Enseñanza Secundaria. El estatuto establecía derechos, obligaciones, formas de acceso a los cargos, mecanismos de ascenso, entre otras disposiciones. La tercera normativa fue el Escalafón docente que establecía la estructuración de la carrera en grados y disponía sueldos y cargas horarias en cada uno.

Este trípode normativo dio una nueva estructura jurídico-administrativa al organismo creado en 1935, pero también contribuyó a definir aspectos identitarios de la institución y, particularmente, de los docentes de Enseñanza Secundaria. Al mismo tiempo que se establecieron garantías jurídicas y se instituyó un sistema de incentivos para la acción de los docentes en tanto trabajadores públicos, se establecieron características propias de la nueva institución, procurando dar respuesta a las necesidades de adecuación del sistema a la realidad. Las Asambleas de Profesores, previstas en el Estatuto del Profesor, fueron uno de los resultados de este proceso de institucionalización y fueron características del funcionamiento de Secundaria hasta 1971.

Este proceso se produce paralelamente a un importante crecimiento de la matrícula estudiantil, un fenómeno que impulsó el crecimiento del organismo.

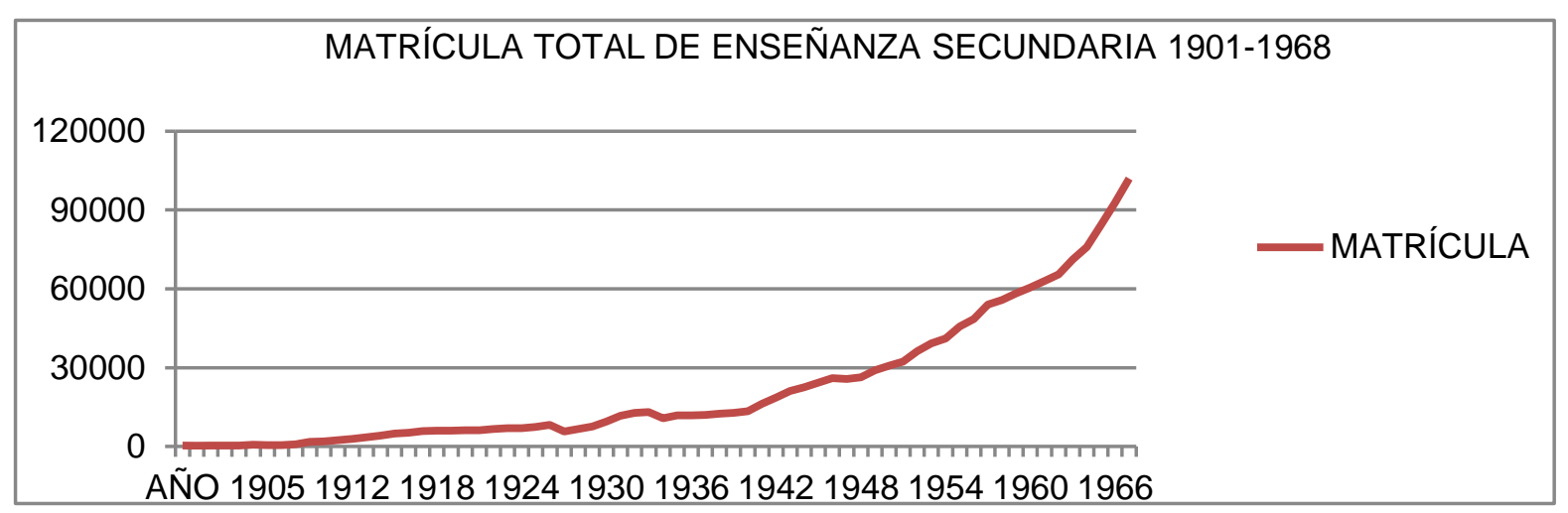

Figura 1 - Matrícula total de enseñanza secundaria (1901-1968).

Fuente: Elaboración propia en base a datos de Consejo de Educación Secundaria, 2008.

1958 que, sin embargo, consagró la autonomía y el gobierno de los órdenes universitarios sin participación del Poder Ejecutivo (MARKARIAN; JUNG; WSCHEBOR, 2008a).

8 Ley 9.691.

${ }^{9}$ Ley 11.285. 
Las Asambleas de Profesores fueron parte de este proceso de expansión y consolidación de una institucionalidad específica. Por un lado, el profesorado se masificaba, y por otro la tarea de enseñanza se enfrentaba a nuevos desafíos: debía adecuarse a la nueva realidad de la masificación y a las nuevas dinámicas económicas, sociales y también culturales que se venían instalando desde las primeras décadas del siglo. Este es el trasfondo de todo el debate pedagógico sobre la enseñanza media: cómo resolver la transición de un modelo universitario a uno de escolarización masiva ${ }^{10}$.

Las Asambleas fueron caja de resonancia de los desafíos profesionales de la tarea docente, pero también ofrecieron la posibilidad de acción colectiva de un cuerpo docente que se estaba conformando, en una institución con serias carencias desde el punto de vista organizativo y de funcionamiento en los aspectos laborales ${ }^{11}$. Las Asambleas compartirían una doble condición: técnica y corporativa.

\section{Antecedentes de las Asambleas de Profesores de Enseñanza Secundaria}

\subsection{La disposición legal de las Asambleas}

Las Asambleas de Profesores de Enseñanza Secundaria fueron un ámbito previsto en el Estatuto del Profesor de 1947 (artículo 40 de la ley 10.973). Allí se establecía: "El Consejo convocará periódicamente, por lo menos cada dos años, a Asamblea de Profesores de los Institutos y Liceos de su dependencia. Estas Asambleas tendrán derecho de iniciativa y función consultiva en los problemas técnico-pedagógicos de la Enseñanza Secundaria."

El artículo en cuestión se inserta en una norma que regulaba el funcionamiento de la carrera de los funcionarios docentes del Consejo Nacional de Enseñanza Secundaria en tanto funcionarios públicos: derechos, obligaciones, formas de acceso a los cargos, mecanismos de ascenso, entre otros. La norma organizaría burocráticamente el trabajo docente en los liceos e institutos dependientes del Consejo, procurando superar un período en que se habían denunciado diverso tipo de irregularidades y déficit en los mecanismos de designación de cargos, fruto de decisiones arbitrarias ${ }^{12}$.

Sin embargo el primer proyecto de Estatuto data de 1937 y había sido elevado al Poder Legislativo por parte del presidente Gabriel Terra y de su ministro de Instrucción, Eduardo Víctor Haedo. En esa oportunidad el Poder Ejecutivo había hecho suya una propuesta elaborada por el propio Consejo de Secundaria ${ }^{13}$ dos años después de iniciar sus funciones. El proyecto fue retomado recién hacia 1946, año que ingresó nuevamente a la Cámara de Senadores en el último año de la legislatura ${ }^{14}$. Finalmente resultó aprobado en el año siguiente, cuando en la nueva legislatura la Comisión de Instrucción de la Cámara de Representantes elevó una propuesta basada en el proyecto de 1937 con un amplio

\footnotetext{
10 Ver antecedentes de esta tensión desde fines del siglo XIX en Oddone; Paris (2010).

11 Entrevistas a Roque Faraone y Ricardo Vilaró (2016); Yaffé (2006).

12 La "ley vendrá [...] a sustituir con el orden a la anarquía actual". (Informe de la Comisión de Instrucción Pública del 12 de setiembre de 1946, en: Diario de Sesiones, Cámara de Senadores, tomo 183, sesión $7 / 10 / 1946)$.

${ }^{13}$ Nota del 24 de abril de 1937 elevando el proyecto de Estatuto del Profesor, firmada por Terra y Haedo. (DIARIO DE SESIONES, Cámara de Senadores, tomo 183, sesión 7/10/1946).

14 Diario de Sesiones, Cámara de Senadores, tomo 183, sesión 7/10/1946.
} 
acuerdo entre los sectores partidarios representados en el Parlamento, el Consejo de Secundaria de la época, la Asociación de Profesores, inspectores y docentes ${ }^{15}$.

En el proyecto de 1937 la convocatoria a asambleas de profesores estaba formulada de la siguiente manera: "Siempre que lo estime necesario, el Consejo podrá formar Asambleas de Profesores de determinadas asignaturas o grupos afines con carácter consultivo para dictaminar sobre los puntos que fije el Consejo. Estas Asambleas deberán expedirse en los plazos que fijarán en cada caso las convocatorias" (artículo 60) ${ }^{16}$. En el proyecto elevado por la Comisión de Instrucción Pública a la Cámara de Senadores en setiembre de 1946 no aparecía ninguna referencia en el articulado sobre las asambleas de profesores, pese a que la Comisión había partido del proyecto anterior que sí lo tenía. En la discusión en la Cámara, el senador Haedo propuso incorporar un artículo sobre las asambleas con la redacción que finalmente resultó aprobada el año siguiente. Ni en el tratamiento del Senado en 1946, ni en el tratamiento de la Cámara de Representantes en 1947 este artículo fue objeto de discusiones. La intervención de Haedo el 7 de octubre de 1946 es la única intervención registrada en las actas parlamentarias que permite vislumbrar el espíritu que sostenía la disposición. En su intervención Haedo argumentó:

El propósito que anima a este artículo es de establecer la obligatoriedad de que
cada dos años, lo que podría llamarse la fuerza viva de la enseñanza, que son los
profesores, se reúna obligatoriamente, en los liceos y en los institutos o todos juntos
en la capital, a fin de vigorizar la marcha de la enseñanza por medio de iniciativas y
observaciones.
Yo creo [...] que es muy útil poner a las asambleas de profesores, no en pugna con
las autoridades, sino en decidida colaboración con ellas y para esto en vez de de
dejarlo librado exclusivamente a la voluntad personal, al esfuerzo de determinados
grupos, es mucho mejor que todos tengan acceso a deliberaciones en las cuales se
entere a las autoridades de todas las iniciativas, de todas las preocupaciones, no
sólo por la enseñanza, sino por la educación, por los medios de impartirla, llegán-
dose, inclusive, a la propia crítica de los procedimientos, o indicando los sustitutivos,
de modo que la autoridad pueda estudiarlos y aprobarlos si los cree conveniente.
(DIARIO DE SESIONES, Cámara de Senadores, tomo 183, sesión 7/10/1946).

La segunda redacción del artículo le otorgaba mayor consistencia al nuevo ámbito al establecer una periodicidad a las convocatorias $y$, sobre todo, al establecer la responsabilidad del Consejo en convocar a las asambleas, siendo que en la versión de 1937 esta era una prerrogativa de ese órgano, pero no constituía una obligación. Asimismo, la fórmula "tendrán derecho de iniciativa y función consultiva en los problemas técnicopedagógicos", que será objeto de interpretaciones posteriores en el seno de la Asamblea, consignaba derechos propios del organismo que se creaba.

Las fuentes disponibles no permiten avanzar demasiado en el alcance que atribuían

15 El Informe de la Comisión de Instrucción Pública de la Cámara de Senadores de setiembre de 1946 destaca
el aporte del Consejo, de la Asociación de Profesores, de los inspectores de Secundaria y, especialmente,
de Dardo Regules por su colaboración en la redacción. (Informe de la Comisión de Instrucción Pública del
12 de setiembre de 1946 , en: Diario de Sesiones, Cámara de Senadores, tomo 183 , sesión $7 / 10 / 1946$ ). Esto
también es destacado por el senador Zavala Muñiz que ofició de miembro informante de la Comisión de
Instrucción Pública del Senado en la Cámara (DIARIO DE SESIONES, Cámara de Senadores, tomo 183,
sesión 7/10/1946) y en el Informe de la Comisión de Instrucción Pública de la Cámara de Representantes
en 1947 y en la intervención del miembro informante, el diputado Quilici. (DIARIO DE SESIONES, Cámara
de Representantes, tomo 471, sesión 6/8/1947).
16 Proyecto contenido en los antecedentes elevados a la Cámara de Senadores en setiembre de 1946 por
parte de la Comisión de Instrucción Pública. (DIARIO DE SESIONES, Cámara de Senadores, tomo 183,
sesión 7/10/1946). Hist. Educ. (Online) Porto Alegre v. 22 ก. 55 maio/ago. 2018 p. $38-60$ 
a las asambleas los actores que promovieron la inclusión de esta disposición en el Estatuto. Haedo anticipó la eventualidad de la crítica y la revisión de los procedimientos y estableció como necesaria la colaboración entre asambleas y autoridades, en caso de un eventual conflicto.

\subsection{Congresos y reuniones}

Sin el carácter formal que adquirirían las Asambleas previstas en el Estatuto del Profesor, habían existido previamente una serie de convocatorias a actividades de diverso tipo, organizadas por diferentes entidades vinculadas a Secundaria. No necesariamente todos estos eventos representan una secuencia de un mismo proceso, ni las asambleas de profesores que serían convocadas a partir de 1949 son su continuación en nuevo formato institucional. Pero es pertinente revisar estas instancias, identificar sus parecidos y continuidades, así como los reconocimientos expresos de antecedentes entre unas y otras.

El propio Consejo, por iniciativa del Director Armando Acosta y Lara en 1943 convocó a un Congreso Nacional de Profesores de Enseñanza Secundaria. La organización recayó en una comisión presidida por Antonio Grompone, entonces Decano de la Facultad de Derecho y luego primer director del Instituto de Profesores. En la presentación del Congreso en el número 15 de la revista Educación y Cultura se indicaban como antecedentes dos congresos nacionales de profesores organizados por la Asociación de Profesores de Enseñanza Secundaria y Preparatoria del Uruguay en 1925 y $1934^{17}$, el Primer Congreso Nacional de Directores de Liceo en agosto de 1936, que habría sido precedido de reuniones de Directores de Liceo durante los decanatos de Lapeyre, Cornú y Musso ${ }^{18}$. También se enumeraban los denominados Cursos Suramericanos de Vacaciones de enero de 1938, dedicados a evaluar el plan de estudios de $1937^{19}$.

La diferencia de la convocatoria de 1943 era su carácter "oficial" al ser convocado

\footnotetext{
17 La Asociación de Profesores de Enseñanza Secundaria y Preparatoria del Uruguay continuó organizando Congresos aunque no con regularidad bienal. Tenemos noticia de la realización del " $V$ Congreso Nacional de Socios" en la primavera de 1953 en La Paloma. El temario estuvo dedicado a "aspectos sustanciales de la Reforma". En el Boletín que comunica las actuaciones del Congreso fue denunciada la falta de interés de la mayoría de los profesores ante instancias de consulta impulsadas por la Asociación, que impidió que hubiera un pronunciamiento expreso sobre muchos de los puntos que requerían consensos amplios. Si bien se trataba de un ámbito gremial y profesional, el Consejo colaboró en la organización y estuvo presente el Director General Ruggia. (ASOCIACIÓN DE PROFESORES DE ENSEÑANZA SECUNDARIA Y PREPARATORIA DEL URUGUAY, 1954).

18 En 1951, puestas en marcha las Asambleas de Profesores creadas en el Estatuto, el Consejo convocó a un Congreso Nacional de Directores de Institutos y Liceos Oficiales de la República en el que participaron también autoridades e inspectores. El temario fue sumamente amplio, abarcando desde aspectos de funcionamiento de los institutos, hasta cuestiones organizativas mayores. Se habría reiterado una estructura de comisiones, incluyendo una destinada a "asuntos no previstos" y otra "de iniciativas". (CONSEJO DE EDUCACIÓN SECUNDARIA, 2008). En la Memoria del Consejo correspondiente al período 1948-1955 se refiere a este evento como el Primer Congreso Nacional de Directores de Institutos y Liceos Oficiales de la República y se informa escuetamente de su realización al final del apartado destinado a informar el reglamento de las Asambleas de Profesores aprobado en 1949 y la realización de las dos primeras Asambleas. El Congreso de Directores habría tenido un funcionamiento análogo al de las Asambleas de Profesores.

19 Otras referencias a este evento no establecen que su objetivo se haya reducido a la evaluación del Plan. Por un lado se lo presenta como una convocatoria que reunió personalidades de países de la región, cuya organización también tuvo a Salterain Herrera como protagonista, presidiendo la comisión organizadora designada por el Ministerio de Instrucción Pública Haedo. (CONSEJO DE EDUCACIÓN SECUNDARIA, 2008, p. 78).
} 
por las autoridades. El temario incluyó la discusión acerca del cumplimiento de los fines establecidos para la enseñanza secundaria en la ley de 1935, si se debía incluir además de una formación general la preparación técnica, los ciclos en que debía dividirse este tramo educativo, la coordinación con los demás niveles, los resultados de los estudiantes y la eficiencia del liceo para la formación integral de adolescentes varones y mujeres ${ }^{20}$. Tanto el carácter oficial como las temáticas abordadas presentan líneas de continuidad con las primeras convocatorias a Asambleas de Profesores, una vez vigente el Estatuto del Profesor.

Pero existieron otras convocatorias de diferente alcance, que también dan cuenta de una cultura que valoraba la deliberación y la organización de ámbitos de intercambio entre el profesorado. En el trabajo coordinado por el historiador Benjamin Nahúm (CONSEJO DE EDUCACIÓN SECUNDARIA, 2008) se reseñan otros eventos en el período previo a la instalación de las Asambleas de Profesores, por ejemplo, la "Concentración Nacional de Profesores Liceales" de 1937 convocada por el Consejo para discutir la implementación del nuevo plan de estudios impulsado en la primer etapa de existencia autónoma del consejo o un "Congreso Nacional de Educadores" convocado en 1945 por el Comité Nacional de Educadores, un ámbito que buscaba tener representación gremial amplia de los docentes de los diferentes niveles educativos a través de organizaciones que los nucleaban.

Esta enumeración refleja la existencia de cierta cultura de reunión y deliberación de parte de los actores docentes de Secundaria, siendo este tipo de reuniones una herramienta de la propia institucionalidad para tramitar o discutir propuestas. Estas características prefiguraban condiciones favorables a la instalación de un organismo como las asambleas de profesores. También se podría incluir en el recuento iniciativas de este estilo en momentos muy diferentes de la historia educativa del país o de otros ámbitos educativos. En tal sentido podría mencionarse una importante tradición de congresos de maestros del nivel primario, pero más cercano al nivel medio, la tradición de los claustros universitarios tempranamente desarrolló estructuras también asamblearias ${ }^{21}$.

\section{Proceso de consolidación e institucionalización de las Asambleas}

En el período que estudiamos se produce la instalación y primera consolidación de las Asambleas de Profesores, o "Asambleas Artículo 40" como eran denominadas coloquialmente, en referencia a la normativa que les dio origen. A lo largo de las seis primeras reuniones, se produjeron algunos cambios significativos en materia organizativa, que acompañaron el creciente lugar que tuvieron en la estructura interna del Consejo. En este período las Asambleas se convirtieron en ámbitos de discusión de los temas centrales de Secundaria. Además, la Asamblea y algunos de sus órganos, se constituyeron en interlocutores de primer orden ante el Consejo. De acuerdo a la información disponible actualmente, en el período 1949-1971 las Asambleas habrían tenido 15 sesiones entre

${ }^{20}$ El Congreso Nacional de Profesores de Enseñanza Secundaria. Educación y Cultura - Revista Uruguaya de Información Pedagógica, n. 15, nov./dic. 1942.

${ }^{21}$ Exagerando el argumento, en las primeras iniciativas de organización de un sistema educativo nacional de masas, pueden rastrearse ideas de este tipo. En un proyecto de ley de educación elaborado por Juan Vedia en 1873 se incluía como una de las disposiciones "la realización regular de asambleas de maestros". (BRALICH, 1987, p. 56). 
La forma de participación y la organización interna fueron cuestiones que se dirimieron hacia la tercera Asamblea y que condicionaron el devenir posterior del órgano. Ya la segunda instancia, convocada seis años más tarde que la primera, introdujo un mecanismo de representación de los docentes de cada liceo. Este fue un cambio significativo si se considera que la primera convocatoria fue abierta, pudiendo participar todo docente que así lo deseara. Arturo Rodríguez Zorrilla, en una alocución al cierre de la quinta Asamblea se referió a la Primera como "desordenada" y "tumultuosa": "nos dejó un amargo recuerdo, una amarga sensación de frustración" (V ASAMBLEA DE PROFESORES, 1961). En la instancia de 1954 se habría discutido la necesidad de un mecanismo de representatividad, pero también sobre cómo aplicarlo para que fuera efectivo. El régimen de suplencias también habría sido objeto de controversias. En la instalación de la Tercera Asamblea, el miembro informante de la Comisión Organizadora, Juan Carlos Arruti, informó de un conjunto de avances en materia organizativa: la iniciativa de la tercera convocatoria había sido de un grupo de docentes en diciembre de $1958^{22}$, el Consejo fue receptivo y puso a disposición recursos para la organización, se procuró la continuidad con la Asamblea anterior a partir de sus actas, de dispuso de viáticos para la participación de profesores del interior. Quizá lo más sobresaliente fue la participación de un conjunto de docentes en la Comisión Organizadora junto al Consejo. Este giro es clave para comprender la naturaleza de un órgano que forma parte de la estructura del Consejo, al tiempo que participan de su organización y gestión los docentes en su condición de tales.

La primera Asamblea ya había aprobado un reglamento interno con 24 artículos que fue ratificado en la segunda. El texto establecía la existencia de una Mesa de la Asamblea y regulaba la mecánica de deliberación y resolución. No establecía instancias preparatorias ni posteriores a la sesión de la asamblea. Sin embargo creaba un mecanismo de comunicación con el Consejo para dar continuidad a las discusiones: "La Mesa de la Asamblea, una vez terminadas las sesiones, elevará de inmediato al Director General todos los antecedentes a fin de que puedan ser publicados o considerados por el Consejo." (3a ASAMBLEA DE PROFESORES, s/d a). Otro documento anterior a la tercera Asamblea incluyó un reglamento de 15 artículos con algunas variantes. Este tenía un carácter más general: reglamentaba la elección de delegados por liceo mediante voto secreto a través de listas con lemas y sublemas, establecía una Comisión Organizadora (Coap) designada por el Consejo, facultaba al Consejo a definir el temario recibiendo sugerencias de la Coap y del profesorado, reglamentaba la forma en que se recibirían trabajos de todo profesor que desee hacerlo y establecía la creación de comisiones para cada uno de los temas a tratar, consagrándolas como una instancia central de tratamiento en profundidad de los temas para elevar informes a las sesiones plenarias. El reglamento también establecía que se debían comunicar las posiciones minoritarias tanto en las comisiones como en la asamblea.

La discusión de aspectos reglamentarios del funcionamiento insumió una importante dedicación en la tercera asamblea en 1959. Dada la naturaleza del dispositivo, el establecimiento de procedimientos y garantías era fundamental en la construcción de su

\footnotetext{
22 Este impulso es coincidente en el tiempo con la movilización de docentes y estudiantes universitarios, con apoyo del movimiento sindical para promover la aprobación de la Ley Orgánica de la Universidad que consagraba la autonomía y el cogobierno. La Ley fue aprobada en octubre de 1958. Ver: Markarian; Jung; Wschebor (2008a).
} 
andamiaje.

Para tener una idea del alcance que tuvieron las asambleas en términos de participación se infirieron algunos datos al respecto. De las Nóminas de Delegados a la cuarta Asamblea, surge que estaban habilitados para participar en calidad de delegado 179 profesores de Montevideo y 135 del interior. (IV ASAMBLEA NACIONAL DE PROFESORES, 1960a). En una votación en la quinta Asamblea se contabilizaron 132 votos de la capital y 89 del interior. (V ASAMBLEA DE PROFESORES, 1961). Si bien no podemos precisar el número total de docentes hacia esas fechas, en 1935 el número habría llegado a 1500. (CONSEJO DE EDUCACIÓN SECUNDARIA, 2008, p. 33).

Una vez instalados los mecanismos de representación y ordenada la mecánica de funcionamiento de las instancias deliberativas, el proceso de institucionalización estuvo pautado por la creación de un órgano que diera continuidad al trabajo de las sucesivas reuniones, produjera insumos y funcionara como portavoz de la Asamblea. Un antecedente era la resolución de la segunda Asamblea de crear una comisión que ordenara los trabajos recibidos, recibiera nuevos y remitiera a los liceos toda la documentación. Como tal, la idea de una comisión de funcionamiento permanente estaba incluida en algunos insumos presentados por los docentes de varios liceos a la tercera Asamblea, como una "medida tendiente a la consolidación de la Asamblea". A modo de ejemplo, la propuesta del Liceo de San José le asignaba como cometidos: informar lo actuado al Consejo, obtener del Consejo el cumplimiento de lo resuelto por la Asamblea, exigir cumplimiento de leyes y reglamentos, convocar a la Asamblea cuando el Consejo no lo haga en tiempo, preparar material, estudios, proyectos, hacer publicaciones, convocar extraordinariamente (por unanimidad de sus miembros) a la Asamblea por hechos urgentes. El tratamiento de estas propuestas fue priorizado en 1959 cuando se aprobó la creación de una Comisión Permanente y un régimen de funcionamiento anual de la Asamblea. Estas iniciativas se incluyeron en un nuevo reglamento aprobado el 5 de noviembre de 1959 por el Consejo (circular no 751). El nuevo reglamento sin embargo mantenía la Comisión Organizadora de la Asamblea de Profesores (Coap) designada por el Consejo e integrada por nueve miembros de los cuales cinco eran propuestos por la Comisión Permanente. El mecanismo de representación establecía la participación del $8 \%$ de los docentes de cada liceo, reglamentando todo el procedimiento de elección a cargo de la dirección de cada instituto. Además se establecía que los inspectores y directores integraran la Asamblea con voz y se aprobaban otras disposiciones que en muchos casos confirmaban el esquema prefigurado: todo docente podría presentar iniciativas, la Asamblea aprobaría su reglamento interno, el temario lo definiría el Consejo y la propia Asamblea dentro de los límites legales del Estatuto que las creaba. (4a ASAMBLEA DE PROFESORES, s/d).

El profesor Roque Faraone, secretario de la mesa de la III Asamblea recuerda que el desconocimiento del Consejo presidido por Coelho de Oliveira a una petición de la II Asamblea fue determinante para que los delegados de los instituto Vásquez Acevedo y Batlle y Ordóñez manifestaran al Consejo la preocupación por la organización de la Asamblea para su buen desarrollo ${ }^{23}$. En la preparación de la misma estos profesores fueron integrados a la Comisión Organizadora del Consejo y se garantizaron los aspectos mínimos de funcionamiento. Durante el transcurso de la Asamblea una delegación se entrevistó con

23 Entrevista con Roque Faraone (2016). 
el Consejo requiriendo la aceptación para la creación de un órgano que entendiera entre los períodos de sesiones. Faraone protagonizó una negociación con el Director Batlle Vila que finalmente dio cabida a la propuesta. En esas conversaciones se habría planteado el malestar de un número importante de delegados que habría estado dispuesto a declararse en sesión permanente en caso de no ser reconocida la comisión permanente por parte del Consejo.

Faraone afirma que en torno a la III Asamblea se nucleó un grupo de profesores jóvenes con inquietudes renovadoras acerca del funcionamiento del Consejo. El programa incluía un funcionamiento transparente y racional de la administración, impulso a la profesionalización y a la formación del profesorado, entre otros. Si bien eran personas generalmente identificadas con la izquierda, las ideas que primaron referían a la racionalización de la gestión de Secundaria; incluso los aspectos pedagógicos habrían estado en un segundo plano. Este grupo impulsó una lista en las siguientes elecciones al Consejo bajo el lema "Nueva Secundaria" y fue protagonista del proceso de conformación de la Federación de Profesores que instaló un ámbito de funcionamiento sindical hasta entonces inexistente ${ }^{24}$.

En abril de 1959 el Consejo le otorgó a la Comisión Permanente una oficina en el Instituto Batlle y Ordóñez y dispuso de fondos para algunas actividades (traslados desde el interior, impresiones). En la solicitud presupuestal anual el Consejo incluyó rubros para la Comisión y la Asamblea. Sin embargo el apoyo recibido fue considerado insuficiente. (COMISIÓN PERMANENTE, s/d).

La Comisión Permanente constituyó un punto de inflexión. Además de garantizar el adecuado funcionamiento de las instancias deliberativas que había reclamado el grupo de profesores más activo en aquella instancia, instaló un ámbito que rápidamente fue reconocido como interlocutor por parte del Consejo. También generó una estructura de subcomisiones que permitieron elaboración de insumos y sistematización de documentos e información que serían volcados a los plenarios. Esto explica en buena medida el avance en la producción de documentos y de las propias reuniones, aunque deben considerarse también algunas condiciones generales de Secundaria y del país. Por ejemplo, a lo largo del período se intensificó la elaboración sobre las características de la reforma que debía tener la enseñanza secundaria. Para comprender la profundización en las propuestas de reforma no alcanza con reconocer las capacidades internas que desplegó la Asamblea a través de su institucionalización, debe considerarse además el papel central otorgado a las Asambleas por el Director General Alberto Rodríguez designado en 1960.

\section{Alcances y naturaleza de las Asambleas}

El alcance de los pronunciamientos de las asambleas de profesores y su propia naturaleza fueron también objeto de interpretación en las primeras reuniones. El texto legal establecía "derecho de iniciativa y función consultiva en los problemas técnico-pedagógicos de la Enseñanza Secundaria”. La primera delimitación era la materia: problemas técnicopedagógicos de la Enseñanza Secundaria, es decir no se trataba de un ámbito gremial o sindical. La especificidad técnico-pedagógica distanciaría a las Asambleas de Profesores de otras modalidades de participación de docentes en ámbitos formales del sistema

24 Entrevista con Roque Faraone (febrero de 2016). 
educativo que tenían precedentes. Una modalidad de participación era la de elección de algunos miembros del Consejo de Enseñanza Secundaria que eran elegidos mediante elección con voto secreto por parte de todos los docentes. Luego los consejeros electos actuaban en tanto miembros de un órgano colegiado de dirección y formalmente a título personal. Otra modalidad había sido ensayada en la Universidad. Si bien la Ley Orgánica de 1958 consagraría el cogobierno pleno de los órdenes docente, estudiantil y de egresados, existía una larga tradición de integración de claustros y consejos por parte de los docentes universitarios. Las Asambleas parecen consagrar otra modalidad de participación, tanto por su carácter técnico como por su funcionamiento deliberativo, es decir, no resolutivo.

La segunda condicionante era la naturaleza de lo que allí se "aprobaba". No se trataba de resoluciones con efectos directos sobre la política educativa o la gestión de Secundaria, sino de pronunciamientos. Las Asambleas permitirían conocer "la opinión del profesorado". En este sentido, era un canal de construcción y manifestación de una voz corporativa. Y para ello se diseñó un sistema complejo de garantías para la participación y la representación, definiendo en detalle mecanismos eleccionarios y formas de funcionamiento que permitieran dejar constancia de las posiciones minoritarias.

El tercer parámetro estaba dado por la relación con el Consejo, es decir las autoridades de Enseñanza Secundaria. En esto radicaba la particularidad de la Asamblea de Profesores. En la medida en que integraba el organismo, tenía un carácter institucional. Pero para el ejercicio de su asesoramiento técnico al Consejo debía tener márgenes de autonomía para posicionarse. El Consejo, por su parte, tenía la posibilidad de condicionar el accionar de la Asamblea a través de diversas vías: la definición del temario sobre el que se consultaba a la Asamblea; el otorgamiento de recursos para funcionar, especialmente para producir insumos, dar seguimiento a los temas, realizar las convocatorias con la información necesaria y garantizar la participación de docentes de todo el país; la consideración de las propuestas y posiciones emanadas de la Asamblea en las decisiones que tomara.

Se delineaban dos características fundamentales del dispositivo. Por un lado el carácter colectivo y deliberativo de la producción técnico-pedagógica de los docentes. Por otro lado la institucionalización de la voz de los docentes en tanto cuerpo docente.

Al respecto del alcance y la naturaleza de las Asambleas se han identificado posiciones divergentes entre los propios protagonistas y a lo largo del tiempo. Habrían existido dos grandes tendencias. Un grupo se propuso avanzar en las competencias de la Asamblea. Otro sector, minoritario, se manifestó favorable a limitar las atribuciones. Ambas posiciones disputaron el significado del texto legal que disponía la realización de las asambleas. La posición mayoritaria se preocupó de reforzar la capacidad de iniciativa de la asamblea en el temario, incluir funciones de asesoramiento en el gobierno de la institución, intervenir en el estudio de propuestas experimentales a la par del Instituto de Profesores, entre otras. En esta línea el Director General Alberto Rodríguez, promotor de importantes cambios introducidos especialmente a partir del Plan Piloto de 1963, plantearía:

Estas Asambleas, que vienen adquiriendo cada vez mayor importancia, serán en lo porvenir, seguramente, las que regirán los destinos de Enseñanza Secundaria. La regirán por el imperativo de sus mandatos, porque serán ellas quienes establecerán cómo y en qué manera se podrá organizar la enseñanza en el país y cuál es la 
orientación que la misma debe seguir; son estas Asambleas las que habrán de indiciar los distintos métodos y procedimientos para modernizar la enseñanza y para hacerla cada vez más práctica y eficiente. (V ASAMBLEA DE PROFESORES, 1961).

La otra tendencia identificada se manifestó como reacción a algunas de las posiciones anteriores, proponiendo mantener en todo momento el carácter puramente asesor de la Asamblea. Si bien está expresada en un único documento y minoritario, resulta significativa. Este grupo se manifestó contrario a la existencia de la Comisión Permanente, argumentando que la autonomía consagrada en la Constitución no debía ejercerse mediante "contrapesos internos", sino que debía confiarse en el "contralor legal"; que el cuerpo docente tenía "cierta falta de madurez como órgano; y que la Comisión Permanente implicaba un exceso de potestades en la Asamblea que actuaba como "vice-consejo" al designar subcomisiones con criterios de idoneidad, no siendo ella integrada con el mismo parámetro. (IV ASAMBLEA DE PROFESORES, s/d).

La mecánica que quedó establecida tras la tercera reunión de 1959 perduró en el tiempo en sus rasgos generales. La Asamblea al finalizar su período ordinario de sesiones - que duraban entre una semana y hasta diez días - designaba una Comisión Permanente que a su vez designaba sub-comisiones para tratar temas específicos. Estas subcomisiones generaban informes que servirían de insumos para las reuniones de la siguiente Asamblea. La Asamblea se integraba por delegados de todos los liceos y era convocada por el Consejo que también aprobaba el orden del día. Existía además un mecanismo de incluir nuevos temas, previo a la Asamblea. Para la organización de la Asamblea el Consejo designaba una Comisión Organizadora con miembros propuestos por la Comisión Permanente y por el propio Consejo. La Asamblea al iniciar designaba una Mesa que dirigiría las deliberaciones e integraba una Comisión de Poderes para resolver la situación de los delegados y establecer los participantes y una Comisión de Temario que proponía el temario definitivo. Luego se creaban comisiones por cada uno de los temas y generalmente una comisión para los temas varios sobre los que se recibían ponencias. Las comisiones, que tenían una mesa con presidente y secretarios, analizaban los insumos y producían un informe con propuestas de resolución para la instancia plenaria. Las posiciones minoritarias también eran comunicadas. Finalmente la instancia plenaria consideraba los informes presentados por un miembro informante de la comisión y aprobaba las resoluciones. Al terminar las sesiones, mediante presentación de listas y voto secreto, se elegía a la nueva Comisión Permanente.

\section{La agenda de las primeras Asambleas}

Se identificaron tres conjuntos de tópicos en la agenda del período. El primero incluye el tratamiento de la propia estructura, funcionamiento y organización de la Asamblea, cuyos principales aspectos fueron reseñados en el apartado anterior. El segundo es el que refiere a la reforma de Enseñanza Secundaria. Este fue el gran tópico pedagógico o "significante vacío" (ROMANO, 2010) de la década del sesenta. En torno a él se estructuraron temáticas y aspectos variados: "fines"; planes, ciclos y asignaturas; "coordinación de las enseñanzas", entre otros. Por último hay un tercer conjunto heterogéneo en el que incluimos otros temas específicos. Algunos de mayor alcance como el Estatuto del Profesor o la Ley Orgánica del ente, y otro más acotados pero en algunos 
casos muy significativos para el proceso de institucionalización del Consejo: la organización de la inspección, los liceos nocturnos, las propuestas extracurriculares, los internados para estudiantes. La variedad de temáticas, refleja el papel que le fue otorgando a las asambleas el grupo de profesores que promovió el proceso de consolidación de las mismas.

Si bien la reforma fue un asunto tratado como tal, puede afirmarse que no hubo tema que no guardara relación con éste. Toda discusión sobre la introducción de modificaciones tenía como horizonte la necesidad de reformar la propuesta educativa. Incluso se diferenció tempranamente, en la segunda Asamblea, el tratamiento de las "realizaciones inmediatas", del tratamiento de "la reforma". Las primeras referían a cambios parciales, que no insumían transformaciones en cadena de múltiples aspectos y que no implicaban nuevos gastos. Sin embargo se las entendía como avances hacia un proceso de reforma de carácter más general. Se discutió acerca del carácter permanente de la reforma o de la necesidad de desarrollar cambios continuos para que la reforma no fuera necesaria. La noción de reforma, en cualquier caso, refería a un déficit en el funcionamiento y a la necesidad de adecuación a las exigencias de la realidad social, del adolescente o del ideal de educación que se perseguía.

La estrategia analítica que proponemos no es recorrer cada uno de los asuntos definidos en los temarios. Plantearemos, en cambio, que existieron algunos tópicos que atravesaron distintas discusiones y que resultan significativas para comprender cómo aquellos protagonistas concibieron la realidad educativa de la enseñanza media y configuraron un discurso pedagógico en torno a ella.

\subsection{Condicionantes sociales y culturales}

Los problemas sociales, sin ocupar un lugar central, son un telón de fondo de las discusiones educativos. El foco está puesto en variables culturales, más que sociales. Se habla de "déficit de cultura y de formación" o de "desacuerdo entre la Enseñanza Secundaria y la cultura actual" que se refleja en la deserción. (3a ASAMBLEA DE PROFESORES, s/d b). Estas distancias culturales a veces se planteaban relacionadas al cambio civilizatorio que se estaba sufriendo con los avances técnicos y científicos que afectaban diversas esferas de la vida cotidiana.

Al momento de las respuestas, los aspectos culturales vuelven a aparecer. Tal es el caso de una propuesta de modificación de la enseñanza del idioma español para revertir problemas asociados al aumento de número de estudiantes, la "generalización de lecturas de baja exigencia al lector", la emigración de idiomas neolatinos, entre otras. Se proponía extender la enseñanza del idioma español por su importancia formativa como instrumento de los demás conocimientos y forma de expresión de ideas y defensa de la personalidad. El trasfondo supone cambios sociales, pero la argumentación de la propuesta está hecha en términos educativos y culturales. Se defendía el carácter formativo de la enseñanza secundaria por sobre el informativo (FABRI; GÜIDA, s/d) y la tarea de enseñanza se concebía en términos culturales, rebasando lo estrictamente intelectual:

La enseñanza secundaria debe ser, a mi juicio, fundamentalmente cultural. Cualquiera sea la duración de los estudios debe predominar en ellos lo cultural, entendiendo por esto la educación del adolescente como medio de formación del hombre, no sólo en su extensión intelectual o en el mayor grado de las formas del saber sino en su capacitación para la humana convivencia. (AZZARINI, s/d). 


\subsection{Cultura y trabajo}

Parecería haber un consenso en la necesidad de superar el carácter meramente preuniversitario de la enseñanza secundaria. Por un lado porque el crecimiento de la matrícula y la variación de los perfiles de estudiantes hacía que no todos los egresados ingresaran a la Universidad. Pero además, porque se reivindicaba una especificidad de la Enseñanza Secundaria o de la Enseñanza Media. Esto ponía en discusión la finalidad de esta enseñanza, sus contenidos y su relación con el mundo del trabajo. Parecería generalizada la idea de que era necesario incluir "formación utilitaria" junto con la llamada "formación cultural". La cultura estaba asociada a los estudios desinteresados, a una formación general e integral que permitiría la profundización en los estudios superiores ${ }^{25}$. Por lo tanto la principal novedad radicaba en introducir contenidos que fueran útiles de manera más inmediata.

Hubo propuestas de diferente tipo, por ejemplo, en las discusiones sobre el "Liceo nocturno" con propuestas adaptadas a las necesidades de la población trabajadora en las que la variación no fuera sólo del horario de las clases Otra propuesta innovadora fue realizada por el profesor Juan Carlos Arruti que propuso la creación de "Preparatorios para la actividad civil" y que se incluyeran materias "facultativas de especialización" en los preparatorios vigentes. (ARRUTI, 1960a).

Luce Fabbri representó una posición diferente sobre este asunto. En un documento sobre fines de la Enseñanza Secundaria planteaba: "[...] los dos fines que tradicionalmente se oponen en las discusiones acerca de Enseñanza Secundaria: 'preparar para la vida y 'preparar para ganarse la vida', no difieren sino en amplitud, puesto que el segundo está incluido en el primero". (FABRI, s/d). Esta posición incluía el trabajo como parte integrante -no exclusiva- del horizonte futuro del joven para lo cual debía formar Secundaria; y postulaba una necesidad creciente de formación general debido a los procesos de tecnificación en marcha. Como corolario, la educación técnica era puesta a la altura de la formación profesional, ambas deberían ser posteriores en el tiempo a la Enseñanza Secundaria y ésta debería ofrecer la misma formación general para ambos trayectos. Además de los crecientes requerimientos en los trabajos manuales, Fabri postulaba la disminución de horas de trabajo debido a la tecnificación, lo que redundaría en mayor tiempo libre y por lo tanto mayor necesidad de una "cultura humanística desinteresada"26.

\subsection{El adolescente y la adolescencia}

Las referencias a la adolescencia como la condición específica de los estudiantes en el nivel secundario son recurrentes. Este tipo de afirmaciones tienen dos sentidos: referir a la especificidad de la enseñanza secundaria y plantear los problemas de adecuación de la propuesta formativa ${ }^{27}$.

Horacio Azzarini en el informe ya citado establecía como particularidad de

${ }^{25}$ Ver Batista (2015) para una revisión de la idea de cultura en las posiciones del Grupo Universidad y la Revista Ensayos, que reaccionaron a las posiciones del gobierno y del Consejo en la segunda mitad de la década de 1930.

${ }^{26}$ Además de una notoria inspiración vazferreireana hay una referencia expresa. Las referencias al filósofo Carlos Vaz Ferreira tienen un peso relativo importante en las escasas referencias que se encontraron en los documentos analizados a pedagogos nacionales.

${ }^{27}$ El historiador Juan Pedro Barrán ha señalado para Uruguay la aparición de la figura del adolescente en las primeras tres décadas del siglo XX, como parte del proceso modernizador del novecentos. (BARRÁN, 1996). 
Secundaria que el educando sea adolescente, diferenciándolo del niño. Por lo tanto la enseñanza que se ofreciera no debía ser la continuidad y mera profundización de los conocimientos trasmitidos en la escuela. La adolescencia implicaba una edad específica y problemática. Hay referencias expresas a algo así como una psicología adolescente, generalmente asociada a problemas y conflictos. Al igual que en el documento presentado por Azzarini, otro informe hace notar la "coincidencia" del ingreso a Secundaria con esta etapa vital en que hay una "crisis psicosomática que provoca perturbaciones físicas, mentales, afectivas y sociales". (IV ASAMBLEA DE PROFESORES, 1960b). La "abulia", el "fracaso" o el "desinterés" causaban deserción.

Otras posiciones recurren a las características de la adolescencia pero las inscriben en perspectivas sociales, no estrictamente psicológicas. En una exposición sobre la reforma de Secundaria, Luis Sánchez Roch afirmaba: "se dice que el joven no estudia, que es desaprensivo, y hasta irrespetuoso, etc. Eso es obra nuestra. Esa manifestación no es más que lo que absorbe de la realidad social que lo rodea. Los problemas económicos han hecho del hogar, del Liceo, de la calle, una escuela de desconformidad, de subversión [...] Los valores morales fundamentales, en el orden social y pedagógico, son hoy secundarios, vivimos el triunfo de la medianía". (SÁNCHEZ ROCH, s/d). Desde esta perspectiva los problemas del adolescente no serían más que manifestaciones de una realidad social general en crisis.

El lugar que toma la adolescencia como etapa psicológica conflictiva o el adolescente portador de una cultura juvenil rebelde, desplaza otras posiciones posibles de los estudiantes. Romano (2010, p. 47) ha señalado que luego de 1935 los estudiantes fueron paulatinamente desplazados de cualquier ámbito de cogobierno. Hasta la segunda Asamblea se consideró la propuesta de constitución de una Asamblea del Claustro de Secundaria similaral Claustro universitario, integrada por los tres órdenes. Esta propuesta no volvió a formar parte del temario y, como vimos, la Asamblea de Profesores pasó a ocupar el lugar central de incidencia de los protagonistas del organismo. Hacia la década de 1960 el protagonismo se había reducido a los profesores. El estudiante era principalmente un adolescente al cual educar.

\subsection{Escolarización de la enseñanza media}

Como resultado de la centralidad del adolescente y de la revisión de la finalidad de una enseñanza cada vez más demandada y llamada a cumplir nuevas tareas, se produjo un proceso de escolarización de la enseñanza media. Esto implicó un alejamiento de la lógica universitaria y la incorporación de finalidades, dispositivos y discursos propios de la lógica escolar.

Esto queda de manifiesto en la consideración de dos funciones docentes: la de inspector y la de profesor coordinador. Una propuesta de reformulación general de la Inspección de Secundaria que proponía, entre otras cosas, diferenciar la función de supervisión de los liceos, los directores y los administrativos, de la supervisión de los profesores de asignaturas, establecía que

es más importante que cada inspector sea idóneo en materia de educación, como lo exige la ley, que un profundo conocedor de una asignatura dada [...] Interesa su autoridad moral, su comprensión frente a los problemas que deben encarar los 
profesores noveles, su larga experiencia docente y su capacidad para orientar y estimular, más que su erudición. (ARRUTI, 1960b).

El "profesor coordinador" representaba una novedad para las tareas docentes que eran concebidas a partir de la enseñanza de una asignatura. Se subrayaba que su tarea sería pedagógica interviniendo en la vinculación del adolescente, el liceo y la familia, atendiendo la realidad específica de los adolescentes, complementando las funciones de enseñanza con actividades variadas. En la cuarta asamblea se planteaba que para esta función debía "poseerse esa más intensa vocación de educador que trasciende la enseñanza de una determinada asignatura" (IV ASAMBLEA DE PROFESORES, 1960b) y se reclamaba una capacitación específica en el Instituto de Profesores para esta nueva tarea.

\subsection{Experiencia, información y conocimiento para definir el cambio}

Por último nos interesa anotar otro tópico que se despliega a lo largo de la documentación emanada de las Asambleas y que refiere al lugar de éstas como ámbitos de producción de discurso pedagógico. Nos interesa aquí rastrear las demandas de información y estudio científico de la realidad y sus problemas para establecer una reforma. En algunos casos esta base se planteaba como condición necesaria para una reforma. Al mismo tiempo hubo una tarea que las Asambleas empiezan a cumplir de reconocimiento y sistematización de experiencias particulares que se estaban desarrollando y que encontraban en este ámbito un marco de comprensión más general enel que inscribirse.

Ya la segunda Asamblea, al retomar el texto sobre fines aprobado en la primera reunión, incorporó la necesidad de un estudio científico como punto de partida de toda reforma, que además acompañaría el fortalecimiento de la formación de profesores. Se planteaba la necesidad de un "centro de estudios psico-pedagógicos y de estadística" y de "liceos experimentales". (3a ASAMBLEA DE PROFESORES, s/d c). Estas ideas, con agregados se reiteraron en el tiempo. También en la segunda Asamblea se instaló la idea de "realizaciones inmediatas" como un tipo de transformación diferente de "la reforma". A diferencia de la reforma, estas "se basan en conocimiento de la labor docente". Según esta distinción, el cambio general requeriría de estudio científico, pero el cambio parcial podría tener origen en la experiencia docente.

En 1955, un documento elaborado por el profesor Rodolfo Usera y que habría sido recuperado por la Comisión Permanente hacia 1959, planteaba la necesidad de un "instituto de investigaciones" con autonomía técnica dentro del ente y con funciones de documentación, estudio, investigación, proyección e información. Debía tener diversas reparticiones y estar conectado con la Inspección, el Instituto de Profesores y los Liceos de Experimentación. La referencia que se utilizaba era la del Centre National de Documentation Pédagogique francês. (USERA, s/d).

En la cuarta Asamblea se planteaban como medios para la realización de la reforma: a) "la creación de un Instituto de investigación técnico docente" por parte del Consejo, y b) la flexibilización de las disposiciones vigentes "para hacer posibles los ensayos parciales". El Instituto de Investigación estaría integrado por personas representativas de las diversas tendencias educativas, técnicas o capaces de serlo. Deberían estar dedicadas full-time a la tarea y ser compensadas económicamente. Debería 
tener un centro de documentación pedagógica, un centro de estudios sociológicos, un servicio de estadística educativa y liceos experimentales. La orientación general sería "acorde a las conclusiones emanadas de las sucesivas Asambleas". (IV ASAMBLEA, 1960c).

Arturo Rodríguez Zorrilla se referiría en 1961 a la resolución de la segunda Asamblea al respecto, en los siguientes términos:

\begin{abstract}
De esta lla Asamblea de Profesores [...] datan los comienzos, los principios de la elaboración de esta doctrina de la reforma de la Enseñanza Secundaria que empieza ahora a concretarse ya definitivamente en esta Va Asamblea. Fue en aquella Ila Asamblea donde se puso énfasis en este aspecto del problema: que la reforma de la Enseñanza Secundaria era primero, y antes que todo, un problema de método, de procedimiento, de investigación y de elaboración sosegada y seria, de premisas y de hipótesis, para elaborar posteriormente las conclusiones definitivas que se han de traducir en normas y en realizaciones. (V ASAMBLEA DE PROFESORES, 1961).
\end{abstract}

Entre 1961 y 1963 se definió una propuesta de reforma que comenzó a implementarse con carácter experimental. El mentado instituto de investigaciones nunca vio la luz. En cambio, tras el proceso de consolidación que describimos, las Asambleas se convirtieron en el ámbito de producción pedagógica acerca de la reforma. Entre 1949 y 1961 algunas variaciones se habían procesado: los profesores tomaron protagonismo a través de la especificidad técnica de la materia de las Asambleas y los requerimientos científicos fueron sustituidos por otras modalidades de producción. El aumento de la productividad y la profundización en el tratamiento de los temas por parte de las Asambleas y sus comisiones estuvieron acompañados por el respaldo a este órgano por parte del Consejo y del Director Alberto Rodríguez desde que asumió en 1960. Las Asambleas pasaron a ser interlocutores frente al Consejo y protagonizaron el impulso reformista que, de todos modos, luego quedaría trunco como parte del aumento de la conflictividad y la crisis económica y política de fines de los sesenta y principios de los setenta.

\title{
7. Apuntes para concluir. Paradojas de una estructura sui generis
}

El recorrido realizado nos permite avanzar en algunas líneas de análisis que contribuyan a comprender el lugar de las Asambleas de Profesores en el proceso de reconfiguración institucional de la enseñanza media en el Uruguay a partir de la creación del Consejo Nacional de Enseñanza Secundaria. Entendemos que las Asambleas desempeñaron diversas funciones en este proceso.

Por un lado la estructura de asambleas destinadas a intervenir sobre aspectos "técnico-pedagógicos" de la enseñanza secundaria, naturalmente se constituyeron como un ámbito de circulación de ideas. Los participantes de un ámbito de este tipo son agentes que producen ideas y las ponen en canales de circulación reglados que implican la difusión, el debate, la legitimación de las mismas. La asamblea es entonces en primer lugar, un dispositivo que regula la circulación de las ideas. Pero no se trató de una tribuna abierta en la que confluían diversos agentes que intervenían autónomamente. La inclusión de mecanismos de representación hacia la Segunda Asamblea evidencia una aspiración más allá de la deliberación abierta. Y además las asambleas emitían pronunciamientos. Las Asambleas de Profesores desempeñaron nuevas funciones respecto a los congresos y reuniones que las precedieron. Se convirtieron en un actor de Enseñanza Secundaria, con 
algunas particularidades. En tanto actor colectivo tenía una voz que representaba al profesorado como cuerpo y procuraba incidir en las decisiones y en el funcionamiento del Consejo.

En la medida en que fue una estructura inserta en un sistema de decisiones institucionalizado, las asambleas se convirtieron en un órgano más del Consejo. Su carácter institucional ya prefiguraba esta diferencia, no era un ámbito académico o profesional convocado por asociaciones profesionales o incluso por el propio Consejo, pero con fines puntuales. La convocatoria periódica establecida en el Estatuto le daba un estatus diferente. Sin embargo lo que terminó de otorgarle la naturaleza de actor fue la organización que se le dio en el devenir de las primeras convocatorias. La Comisión Permanente fue un hito en este proceso, pero las controversias relevadas acerca de lo que denominamos "naturaleza" de las asambleas evidencian este cambio de cariz. Se procuró convertir a las asambleas en la voz del profesorado. Una voz que representaba a los profesores en tanto integrantes de un cuerpo especializado en los asuntos de enseñanza en el marco de la estructura institucional del Consejo. Ya no se trataba de una organización profesional que si bien guardaba estrechos lazos con el Consejo y sus autoridades, actuaba en una órbita exterior a la institución, como podía ser el caso de la vieja Asociación de Profesores de Enseñanza Secundaria y Preparatoria con fuerte actuación en el período anterior.

Las Asambleas constituyeron un modelo de organización colectiva novedosa. Se diferenciaba de la agremiación o asociación profesional por su carácter institucional, que a su vez lo asemejaban a las formas universitarias de participación. Sin embargo a lo largo de la primera mitad del siglo XX las discusiones acerca de la participación de los actores en ámbitos de deliberación y gobierno de la Universidad giraban en torno a la inclusión estudiantil. Las Asambleas reforzaban la capacidad de incidencia de los docentes de Secundaria y configuraban una forma de entender los asuntos técnicos del ente a partir de la figura docente. Esto las alejó de las discusiones contemporáneas en la Universidad de la República, donde el cogobierno de docentes, estudiantes y egresados que terminó plasmándose en la Ley Orgánica de 1958 también tenía un fundamento técnico: la conducción de la institución debía llevarse adelante autónomamente de los demás poderes políticos y ser el resultado del diálogo de los protagonistas. Estos protagonistas, en una institución de educación superior, no debían ser solamente los profesores. Pero Secundaria había dejado atrás el modelo de educación superior y el estudiante estaba cada vez más identificado con la figura del adolescente. Esto volvía imposible la conformación un cogobierno con participación estudiantil.

La consolidación de las asambleas se produjo en un período en que también se aprobó el Estatuto del Funcionario Docente y se creó el Instituto de Profesores. Formó parte de un proceso de consolidación de la estructura burocrático-profesional de Secundaria. Sin embargo Secundaria en ese momento todavía estaba lejos de consolidar una estructura burocrática tradicional y de acercarse a una política de profesionalización general de los cuadros docentes. Las asambleas vienen a reforzar el carácter técnico de las intervenciones del profesorado, pero en un contexto institucional en que no se dan las condiciones que tradicionalmente garantizan la autonomía técnica de una burocracia profesional especializada, por ejemplo en la transparencia del acceso a los cargos. Esto había sucedido así tempranamente en la Enseñanza Primaria: titulación obligatoria, carrera docente reglada, acceso competitivo y abierto a cargos, estructura de inspección y cuadros 
intermedios. En Secundaria, en cambio, a posibilidad de configurar una opinión del profesorado y hacerla oír en el proceso de toma de decisiones fue la seña de identidad del proceso de profesionalización.

En suma, podemos afirmar que se trató de una estructura sui generis. Era un ámbito en que circulaban ideas técnico-pedagógicas acerca de los problemas de Secundaria, pero que funcionaba como una caja de resonancia y voz del universo de los profesores al interior de la institucionalidad. Formaba parte de la estructura del organismo, como espacio de participación de uno de sus actores - los docentes - considerados agentes indispensables de las discusiones técnicas. Aspiraban a tener un carácter técnico, no gremial, corporativo o político. Pero su consolidación no estuvo acompañada de los tradicionales mecanismos de fortalecimiento técnico de las estructuras burocráticas del Estado.

En el contexto uruguayo, estamos frente a una rearticulación de la tradición autonomista que recorre la organización del sistema educativo a lo largo de su historia. La autonomía de los órganos de la enseñanza probablemente no tenga una única fuente y haya sufrido variaciones a lo largo del tiempo. El proceso de las asambleas de profesores que estudiamos representaría una variante de esta tradición. La conformación de burocracias modernas ofreció garantías de autonomía respecto de arbitrariedades políticas o económicas, dotando al Estado de capacidades propias para orientar la gestión pública. En Uruguay tuvieron fuerte presencia otro tipo de mecanismos, como la descentralización institucional respecto del gobierno central y los espacios colegiados de dirección que permitían la coparticipación de actores con diversas procedencias. En los organismos de la enseñanza estos mecanismos de autonomía incluyeron, especialmente en la tradición universitaria, a la intervención directa de los docentes como una seña específica. Es así que el profesorado de Secundaria designaba la mitad de los integrantes del Consejo. Sin embargo, el proceso estudiado evidencia que esa modalidad de participación docente habría sido insuficiente para un sector importante del profesorado que logró imponer el modelo de Asamblea de Profesores que describimos.

\section{Referencias}

3a ASAMBLEA DE PROFESORES. 3a Asamblea de Profesores (art 40 - Estatuto del Profesor). Procedimientos preparatorios para su reunión. № 2, $\mathrm{s} / \mathrm{d}$ a.

3a ASAMBLEA DE PROFESORES. 3a Asamblea de Profesores (art 40 - Estatuto del Profesor). Procedimientos preparatorios para su reunión. № $3, \mathrm{~s} / \mathrm{d}$ b.

3a ASAMBLEA DE PROFESORES. 3a Asamblea de Profesores (art 40 - Estatuto del Profesor). Procedimientos para su reunión. Antecedentes II Asamblea, s/d c.

4a ASAMBLEA DE PROFESORES. 4a Asamblea de Profesores (art 40 - Estatuto del Profesor). Información sobre relgamentos, temario, plan de trabajo, ponencias. № 1, s/d.

ARRUTI, Juan Carlos. Ponencia para la IV Asamblea de Profesores. "Creación de los 'preparatorios para la actividad civil' y de materias facultativas de especialización técnica a incorporar en los planes de los cursos preparatorios actuales". Florida, 1960a.

Ponencias para la IVa Asamblea de Profesores. Revisión de la Inspección de Enseñanza Secundaria, 1960b.

ASOCIACIÓN DE PROFESORES DE ENSEÑANZA SECUNDARIA Y PREPARATORIA 
DEL URUGUAY. Revista Oficial, 1954.

AZZARINI, Horacio. Comisión Permanente de la Asamblea de Profesores. Sub-Comisión de reforma. Exposición del profesor Arq. Horacio Azzarini. Fines de la Enseñanza Secundaria, s/d.

BARRÁN, José Pedro. El adolescente, ¿una creación de la modernidad? In: BARRÁN, José Pedro; CAETANO, Gerardo; PORZECANSKI, Teresa. Historias de la vida privada en el Uruguay 2. El nacimiento de la intimidad 1870-1920. Montevideo: Taurus, 1996.

BATISTA, Pía. Reforma de la enseñanza secundaria en el Uruguay de la década de 1930: la revista ensayos y la agrupación universidad. Revista Políticas Educativas, v. 9, n. 1, 2015.

BRALICH, Jorge. Breve historia de la educación en el Uruguay. Montevideo: Centro de Investigaciones y Estudios Pedagógicos, 1987.

CÁMARA DE REPRESENTANTES. Diario de Sesiones, tomo 471, sesión del 6 de agosto de 1947.

CÁMARA DE SENADORES. Diario de Sesiones, tomo 183, sesión del 7 de octubre de 1946.

COMISIÓN PERMANENTE. Informe de la Comisión Permanente a la IVa Asamblea de Profesores, $\mathrm{s} / \mathrm{d}$.

CONSEJO DE EDUCACIÓN SECUNDARIA. Historia de Educación Secundaria 19352008. Montevideo: Consejo de Educación Secundaria, 2008.

FABRI, Luce. Fines de la Enseñanza Secundaria. Exposición de la Prof. Luce Fabbri de Cressatti. Comisión Permanente de la Asamblea de Profesores. Sub-Comisión de reforma, $\mathrm{s} / \mathrm{d}$.

FABRI, Luce; GÜIDA, Inés. Iniciativa para una mayor extensión del estudio del idioma español en Enseñanza Secundaria presentada a la Comisión Permanente de la Illa Asamblea de Profesores, s/d.

III ASAMBLEA DE PROFESORES. Versión taquigráfica de la Sesión Plenaria de la III Asamblea de Profesores, correspondiente al 2 de marzo de 1959, 1959.

III ASAMBLEA DE PROFESORES. Versión taquigráfica de la Sesión Plenaria de la III Asamblea de Profesores, correspondiente al 2 de marzo de 1959, s/d.

IV ASAMBLEA DE PROFESORES. Comisión no 1. Del reglamento del art $\mathbf{4 0}$ de la ley 10973. Informe en mayoría, $s / d$.

IV ASAMBLEA NACIONAL DE PROFESORES. Nómina de delegados electos por los liceos e institutos de la capital y Nómina de delegados electos por los liceos e institutos del interior, 1960a.

IV ASAMBLEA DE PROFESORES. Comisión nํ 5. Informe sobre Profesor Coordinador, 1960b.

IV ASAMBLEA. Informe de la Comisión Reforma, 1960c.

MARKARIAN, Vania; JUNG, María Eugenia; WSCHEBOR, Isabel. 1958: El cogobierno autonómico. Montevideo: Universidad de la República, 2008a.

MARKARIAN, Vania; JUNG, María Eugenia; WSCHEBOR, Isabel. 1908: El año augural. Montevideo: Universidad de la República, 2008b.

ODDONE, Juan; PARÍS, Blanca. Historia de la Universidad de la República. Tomo II. La Universidad del militarismo a la crisis (1885-1958). Montevideo: Universidad de la 
República, 2010.

ROMANO, Antonio. De la reforma al proceso. Una historia de la Enseñanza Secundaria (1955-1977). Montevideo: Ediciones Trilce, 2010.

SÁNCHEZ ROCH, Luis. Notas tomadas por secretaría de la exposición del Prof. Luis A. Sánchez Roch sobre "Reforma de la Ensñanza Secundaria", s/d.

URUGUAY. Ley 10973. Estatuto del Profesor, 1947.

USERA, Rodolfo. Comisión Permanente de la Asamblea de Profesores. Sub-Comisión de reforma "Organización de la reforma de la Enseñanza Secundaria. Contribución del Delegado de los Directores de Institutos y Liceos en la Comisión de Estudios Dr. Rodolfo. C. Usera", 1955.

V ASAMBLEA DE PROFESORES. V Asamblea de Profesores. № 1. Discurso inaugural. Acta de sesión de clausura, 1961.

YAFFÉ, Jaime. El gremialismo docente en Secundaria (1919-1964): antecedentes y proceso fundacional de la Federación Nacional de Profesores. In: BARHOUM, María;

PESCE, Fernando; YAFFÉ, Jaime. Federación Nacional de Profesores (1963-2007). 43 años de lucha por la educación públca y los derechos de sus trabajadores. Montevideo: Fenapes, 2006.

LUCAS D'AVENIA es docente de la Universidad de la República (Uruguay) en la Facultad de Humanidades y Ciencias de la Educación, en el Área de Investigación Histórica del Archivo General y en el Instituto Superior de Educación Física. Doctorando de la Universidad Nacional de Quilmes (Argentina).

Dirección postal: Avenida Uruguay, 1695, 11200, Montevideo, Uruguay.

E-mail: lucas.davenia@fhuce.edu.uy

Recebido em 01 de março de 2018.

Aceito em 21 de junho de 2018. 